\title{
Virtual subjects: Using the Internet as an alternative source of subjects and research environment
}

\author{
MICHAEL A. SMITH and BRANT LEIGH \\ University College of the Cariboo, Kamloops, British Columbia, Canada
}

\begin{abstract}
This paper presents a rationale for why some researchers might consider using the Internet for research by addressing four issues. First, a few unique research opportunities afforded by the Internet are discussed. Second, the utility of using newsgroups and the Telnet protocol is described. Third, Internet research ethical guidelines in five areas are developed. Fourth, the data validity issue is addressed, and previous research using Internet and introductory psychology subject samples is replicated. The samples were similar on 5 of 7 demographic variables but differed in age and sex compositions, and response patterns for the two samples were similar to those reported previously. Finally, some limitations of the Intermet are considered, as are its potentials to fulfill traditional research requirements and its use as an educational aid.
\end{abstract}

The number of people using the Internet, the amount and type of information available, and the capabilities of the Internet are rapidly increasing. This suggests that researchers may find the Internet a useful research tool. It has the potential to serve as an alternative or supplemental source of subjects and research environment for traditional psychological investigations. Moreover, the potential impact of the Internet on human thought and behavior can be investigated. Developing the Internet as a research tool requires prudent consideration of important issues such as the ethical use of subjects, viable research methods, and data validity. We address these concerns by considering four issues. First, we consider some of the unique research opportunities the Internet may afford researchers. Second, we briefly describe two characteristics of the Internet that make it a useful research tool. Third, we propose some appropriate ethical guidelines and discuss some unique data security and validity issues that arise when conducting research on the Internet. We describe a survey study we conducted that used both a traditional sample and an Internet sample. This study will be used to assess the potential validity and generalizability of data collected from Internet subjects. Finally, we will discuss a few limitations and potentials of the Internet. By addressing these issues we hope to begin the process of developing the Internet as a valuable addition to the repertoire of research tools available to researchers.

The authors thank Danalee Goldthwaite, William Roberts, and the reviewers for their helpful comments and suggestions on earlier versions of the manuscript. Correspondence concerning this article should be addressed to M. A. Smith, Department of Psychology, University of Toronto, 100 St. George St., Toronto, ON, Canada M5S 3G3 (e-mail: masmith@psych.utoronto.ca).

\section{Why Conduct Research Using the Internet?}

The value of any new research technique lies not in its capability to examine questions already testable using other methods, but in its ability to offer new opportunities for research, examining questions previously too difficult to answer (Castellan, 1991; Lesgold, 1991) or exploring questions that take advantage of its unique strengths. Using the Internet to conduct research offers several advantages over traditional research practices. (We also address its potential to fulfill traditional experimental research requirements later in this paper.) Because of the broad connectivity of the Internet, it is as accessible as radio, television, or the telephone, but it has a degree of interactivity unavailable to any of these other forms of communication (Hewson, Laurent, \& Vogel, 1996). It allows for the transmission of many different types of communication, such as text, sound, graphics, and live interaction between users, which these other types of communication do not. While these other forms of communication can accommodate one or more of these components, only the Internet accommodates all of them within a single medium of communication.

The connectivity of the Internet offers advantages to a broad range of research endeavors. It can serve as a passive source of data, where researchers search and analyze archival material (Bordia, 1996) and geographically distant databases. It can be used as an active source of data, where volunteers participate in current, real-time data collection without the constraint of having to be geographically proximate to the researcher. A third advantage derives from the fact that the Internet connects many nations. This international connectivity can significantly improve the cost and ease of conducting cross-cultural research. Finally, many experiments are subject to various types of demand characteristics, such as subject and experimenter expectancies (Hewson et al., 1996). Using the Internet as a research en- 
vironment allows for a degree of anonymity to both researchers and subjects, decreasing the influence of such demand characteristics (Esposito, Agard, \& Rosnow, 1984).

There are also social and cultural reasons for conducting research using the Internet. Internet culture is unique. It offers an unprecedented opportunity for people to successfully adopt an alternative persona and to interact with others in ways that are quite different from face-toface or telephonic interactions (Bordia, 1996). Many common cues that people use to aid and interpret communications are not present when individuals communicate through a common computer interface (e.g., visual or auditory cues and the appearance of handwritten text). Understanding the strategies and mechanisms people use to communicate without these important cues can provide valuable insight into social behavior.

Societal changes often accompany changes in the way people interact with each other. If the Internet becomes as prevalent in our daily life as television, social patterns may also change. Understanding its potential impact on human behavior is not only interesting, but also prudent. Every tool has positive and negative implications, and the Internet is no different in this respect. These implications must be understood if we are to limit the disadvantages and promote the advantages. The characteristics of the Internet as a system of communication are well suited to examining these types of questions.

A peripheral, though certainly important, reason for using the Internet as a research environment involves its value to researchers at smaller institutions. Small universities and colleges, with their emphasis on small class sizes and small overall populations, often have insufficient population sizes to maintain active research programs. For example, approximately $40 \%$ of the 350 introductory psychology students at the University College of the Cariboo volunteered as subjects in the winter semester. With three active faculty members and several undergraduate courses with lab components, demand exceeds the supply. It is possible to conduct research, but at a much slower pace than at larger universities. With approximately 22 million (and growing) people using the Internet (Reid, 1994), researchers at smaller universities can potentially access a larger subject population than is locally available and work at a pace comparable to that of researchers at larger universities.

This value also applies to researchers who do not use university students as research subjects. Researchers who are interested in working with specialized populations (e.g., homosexuals, people with physical disabilities, or rape victims) but are located in small population centers have difficulty establishing an adequate subject population. For example, the homosexual community will be considerably smaller in a city of 70,000 than in a city of over 1,000,000. However, the Internet has forums (called newsgroups) that can meet the criteria of many specialized interests or population characteristics and the necessary tools to access them. Therefore, researchers who previously had limited access to some special populations no longer have to be at a disadvantage.
To summarize, using the Internet to conduct research can allow researchers to answer questions previously too difficult, time consuming, or costly to be practical. Furthermore, with more people using the Internet every day, it will be important to learn more about the impact the Internet can have on people's social and personal behavior. Finally, by serving as an inexpensive and efficient method to access potentially large numbers of volunteers, the Internet can also level the playing field for researchers at smaller universities who do not have the same subject resources that larger institutions do.

\section{Computer Networks}

Computer networks have a number of characteristics useful to researchers, some of which have already been incorporated into research designs. For example, they permit the transfer of information between machines designed for specific tasks, separating the tasks of data collection and data analysis (Yost \& Bremner, 1985). They can collect data from multiple simultaneous experimental sessions (Hoffman \& MacDonald, 1993; Polson, Miller, \& Karat, 1981). They can also act as actual research environments to address difficulties associated with specific data-collection techniques (Bordia, 1996; Huff \& Rosenberg, 1989).

However, researchers have not fully exploited the capacity for networks to be distributed over wide areas. Computer networks used as research environments (Huff \& Rosenberg, 1989) or to simultaneously collect data from multiple subjects (Hoffman \& MacDonald, 1993) restrict their scope to local, on site, networks. Using widearea networks, such as the Internet, as a research environment broadens that scope.

The many different wide-area computer networks distributed across Canada and around the world represent a large and easily accessible group of potential subjects. Three common wide-area networks are Bitnet, Internet, and Usenet (Kieley, 1993). These networks support a variety of services and software, and they communicate seamlessly with other networks using standardized Internet protocols (Krol, 1992). Some services are network specific; others are common across two or more networks. For example, both Usenet and Bitnet offer special-interest forums (generically known as newsgroups) in which individuals with common interests share information. They are the Internet equivalent of discussion groups or bulletin boards.

Usenet hierarchically organizes newsgroups into seven broad categories: comp.-groups discussing computer science and related topics; news. - groups concerned with the news network and software; rec.- groups discussing hobbies, recreational activities, and the arts; sci.-groups discussing scientific research; soc.-groups discussing social issues; talk.-groups debating controversial or unresolved issues (e.g., religion); and misc.-groups that do not fit into one of the other categories (Krol, 1992). Another well-known top-level group category is alt. Groups organized under this prefix discuss topics from an alternative point of view (hence the prefix alt.). These broad 
categories are then subdivided into an arbitrary number of increasingly specific subgroups (Kieley, 1993; Krol, 1992). For example, readers of the newsgroup sci.psychology may discuss many different aspects of psychology, but readers of sci.psychology.research restrict discussions to research-related topics. This hierarchical organization allows researchers to select research populations as broadly or as narrowly as warranted.

A second useful feature of wide-area networks is the capacity to directly connect to and interact with a remote host computer. Telnet, an Internet protocol, allows subjects to directly connect with a remote host by typing the command Telnet followed by the domain address of the host computer (e.g., telnet cariboo.bc.ca). Once connected, subjects send keyboard inputs directly to the remote host, over the Internet, and use the computing resources of the remote host in the same way they would use the keyboard to send input to their desktop PC (see Krol, 1992, for a more complete description of the Telnet protocol). This capability allows researchers to conduct research on a single computer with subjects from multiple geographic locations. (The next section discusses why researchers might consider using this method instead of other potential methods.)

The previous two sections outlined why and how researchers might use the Internet as a research tool. However, because the Internet is unlike other research tools or environments, using it as such raises a number of unique ethical issues. The next section develops some ethical guidelines suitable for conducting research on wide-area networks.

\section{Ethical Issues}

The Internet is a unique research environment. Recruiting volunteers must be performed differently, and subjects might not directly interact with the researcher. Therefore, it is necessary to devise methods to ensure that subjects receive fair treatment. The University College of the Cariboo ethics committee that deals with research and other studies involving human subjects has recommended that five areas be given particular attention: subject recruitment, informed consent, the protection of a subject's right to withdraw participation at any time, protecting against subject fraud while maintaining subject anonymity, and data security. This section discusses these five areas and the solutions that the ethics committee considered acceptable.

Subject recruitment. A subject recruitment notice must meet several requirements. First, there must be a reasonable expectation that potential readers of the notice are capable of giving informed consent (i.e., be over 18 years of age). Before posting our recruitment notice, we contacted the newsgroup moderator and asked for subscriber information. If the newsgroup was unmoderated, we posted a prerequest, informing the readers of our intent to post a request and asking readers to make a judgment as to the reasonableness of posting a volunteer notice. This served to prescreen the potential newsgroup and allowed for feedback as to the appropriateness of the re- quest. Generally, readers and moderators appreciate the consultation and are willing to accommodate the request.

The actual recruitment notice needs to include more features than do typical recruitment notices. Newsgroup readers tend to be quite skeptical of nontopical requests and dislike advertisements of any kind because they are often of dubious quality. Therefore, the face validity of the notice needs to be high. Our recruitment notice contained the following features: It mentioned that the moderator considered the request acceptable or that there were no objections from any readers; it contained enough information to allow potential subjects to be fully informed before they participated; it provided the ethical approval code, institutional affiliation, the e-mail address of the researcher, and the mailing address of the ethics committee, so that anyone could direct questions or comments about the research to a third party without the researcher's knowledge; and finally, it included detailed instructions on how to participate.

Informed consent. A reasonable method to obtain informed consent electronically needs to be in place. In traditional research settings, people physically sign an informed consent form to indicate their willingness to participate. This is not practical on the Internet. We developed two procedures to obtain electronic consent. We used the first at the time of our research; we developed the second to address deficiencies found with the first method. We describe both techniques for two reasons. One, because not every researcher may have the capacity to implement the second technique (i.e., due to system limitations), the minimal system requirements of the first technique outlines an acceptable method for obtaining consent. The second method, which requires a more powerful operating system to implement than does the first method, describes an efficient way to obtain consent and to address several other ethical and experimental considerations with a single procedure.

The first technique requires potential subjects to use the Telnet protocol to connect to a remote host computer, entering a password after they read the informed-consent message. Typically, people begin interacting with a computer on the Internet by entering a user name to indicate what account they wish to use. The computer then prompts them for a password. Unless the password is correct, the resources and materials available in that account remain unavailable. We presented the informed consent form after subjects entered the account name but before prompting them for the password. The password was presented to subjects after they had read the informed consent form, which included a statement informing them that by typing the password they were giving informed consent and that they were capable of giving informed consent. The host computer automatically disconnected those entering an incorrect password. Because it was impossible to know in advance who would be interested in participating, this method ensured that only those subjects who had connected to the host computer and read the informed consent form accessed the account. 
While the first method adequately indicated that subjects had given informed consent, it also had limitations. There was no way to keep subjects from participating multiple times, and it is difficult to assign subjects to different experimental conditions. To address these deficiencies, we developed a second, more effective technique. After reading the informed-consent form, a subject entered his/her e-mail address and the host computer sent a computer-generated password for the subject to use to access the experiment. The unique password effectively reduces the potential for subject fraud and can also be used for other purposes. For example, a password generated with a specified set of rules can be used to assign subjects to experimental conditions (discussed in greater detail below).

Subject's right to withdraw. To allow a subject to withdraw at any time without penalty and to maximize the security of research material and data, tradeoffs had to be made. To secure our research material and data, our research was done within a captive account. Captive accounts limit the number and types of computing resources available to users of that account. Within a captive account, the host computer ignores all commands except those set by the system administrator.

One of the primary reasons for using captive accounts is to prevent subjects from breaking out of a computing process. Normally, if users want to stop a process, they use one of several standard break keys (e.g., Ctrl-y, Ctrl-z). This terminates the current process and returns the user to the root directory where experimental materials are generally located. In our captive account, these keys are disabled.

Since restricting permissible actions conflicts with a subject's right to withdraw from an experiment, the experimental software needs to include explicit measures to ensure that subjects can withdraw at any point. To accomplish this goal, instructions on how to withdraw were always present on the screen (e.g., "press 0 to withdraw participation"). If a subject withdrew, the host computer closed the connection and returned that subject to his or her local site.

Subject anonymity and data security. The issue of anonymity and security of data is particularly important in a networked environment, especially when large numbers of individuals have potential access to sensitive material. If subjects inadvertently gain access to software and confidential data, or if hackers deliberately break into accounts to alter experimental programs or data files, or to otherwise disrupt data collection, the validity of any collected data is questionable and anonymity is potentially compromised.

Guaranteeing complete data security in a networked environment is not possible. There are too many ways for individuals intent on breaching security to do so. However, it is possible to minimize the risk for people who participate in good faith not to have their anonymity compromised.

As with any research, some methods of data collection are better than others. For example, when conducting mailsurvey research, survey materials are mailed to selected populations for completion and return. A similar proce- dure for distributing survey materials to targeted newsgroups for completion and return is also possible on the Internet. However, this procedure has several weaknesses. Unlike a traditional mail survey in which an experimenter produces and distributes a fixed number of surveys and has the responsibility of tracking and controlling the material, a survey distributed over computer networks would no longer be secure.

There are several ways that material distributed in this way can compromise the security of experimental materials and the anonymity of subjects. A single keystroke can distribute materials to unintended subjects. Subjects can type an incorrect return address or inadvertently forward responses to someone other than the researcher. These two possibilities can result in misdirected responses and hence a loss of confidentiality for the subject. A researcher also immediately loses control of any material distributed on the Internet.

One of the researcher's primary tasks should be to safeguard a subject's anonymity and data integrity. Requiring subjects to connect to a secured captive account allows greater control over subject responses and options, maximizes control over experimental material, reduces the risk of unauthorized distribution, and allows for greater control over data collection and storage.

The risk of general hacking of computer accounts also needs to be addressed. The availability of software that breaks passwords and gains access to confidential data threatens the anonymity of subjects. There are several ways to minimize the risk of compromising a subject's anonymity. One is to identify data with unique identifier codes known only to the experimenter. This minimizes the risk that a hacker will link a specific data set to a specific subject. Another is to send data to a separate account configured in such a way that it can be accessed only from a specific third account with specific commands. This two-tiered security decreases the probability of security breaches.

\section{Data Validity}

So far, we have discussed why researchers might consider using the Internet for research, some characteristics that make it a potentially useful tool, and some ethical guidelines to ensure the fair treatment of subjects. However, there still remains the issue of data validity. For the Internet to be of use as a research environment, any data collected by researchers must be valid, and there is no guarantee that the data collected using Internet subjects are valid or generalizable to the general population. Conclusions drawn from these data may apply only to that select population of computer-literate people who have some self-interest in participating in the particular research project. Therefore, developing the Internet as a research environment requires the validation of data collected from Internet subjects. We will discuss several factors that could affect data validity: subject fraud, data generalizability, and self-selection bias.

Subject fraud. Conducting research in captive accounts with a single password increases the potential for 
subject fraud. Subjects can complete an experiment as many times as they wish, possibly invalidating any conclusions drawn from the data, since no technique to track and prevent this possibility is in place. To reduce this risk, our software determined the domain address (but not the individual's unique account name) with Digital Command Language (DCL) lexical functions and appended that information to the subject's data.

The presence of multiple data sets from the same domain address suggests two scenarios. Either more than one person participated from that site, in which case all sets of data were legitimate, or a single person participated more than once, bringing into question the validity of all data from that site. However, it is not possible to distinguish between these alternatives by using lexical functions. Therefore, we began our data analysis by checking the data for multiple entries from the same domain address. If any were found, they were removed from any further analysis. This is the most conservative of methods for dealing with this problem.

A more effective strategy for reducing the risk of a single individual's completing an experiment more than once and for reducing the risk of introducing deliberate random error is to assign unique passwords to individual subjects. To accomplish this, a subject signs a consent form by entering his/her e-mail address. The host computer generates a unique password and sends it to that e-mail address. It adds that password and the associated e-mail address to a list of acceptable passwords. After subjects enter the password to gain access to the experiment, the host computer invalidates that password. Furthermore, the host computer issues only one password per e-mail address, preventing the same individual from requesting a new password. Unlike the single-password method, assigning every user a unique password allows multiple subjects from the same domain address to participate.

Data generalizability. While the technique of assigning unique passwords reduces the risk of subject fraud, it does not address the issue of whether data collected from Internet subjects are typical or generalizable to the larger population. We addressed this issue in two ways: (1) We examined population demographics and compared data obtained from Internet subjects with another well-established population (i.e., introductory psychology students), and (2) we replicated a study conducted by Ellis and Symons (1990).

Ellis and Symons investigated the nature and frequency of men's and women's sexual fantasies by surveying 307 students at a state university and a junior college in California. Students completed a pencil-and-paper questionnaire designed to investigate sex differences in sexual fantasies. Hypotheses were derived from modern evolutionary theory. Predicted sex differences were found on a number of characteristics, including the salience of visual images, context, personalization, and emotion.

If the Internet sample has similar demographic characteristics as other established populations, the argument can be made that Internet samples are also representative. Furthermore, replicating previous research using dif- ferent populations, different recruitment procedures, and different experimental settings strengthens the argument that data collected from Internet subjects are just as useful as data collected from more traditional populations. Below we describe our research, compare our results with Ellis and Symon's (1990), and use these results to discuss the issue of data generalizability.

\section{METHOD}

\section{Subjects}

Two populations were sampled. Fifty-six introductory psychology students, randomly selected from the Psychology Department's voluntary subject pool, agreed to participate, and 72 subjects were recruited from the Internet by posting a volunteer request to the moderated newsgroup sci.psychology.research. The listowner previewed the request and approved its posting. This newsgroup had a readership of approximately 10,000 (J. M. Grohol, personal communication, Sept. 26, 1994), with approximately 12 daily contributions.

The Internet recruitment notice followed the ethical guidelines outlined above. It contained a description of the research project, a statement confirming that the human ethics committee approved the project, the project's ethics approval number, and the surfacemail address of the ethics committee. The notice informed potential subjects that the experimental site would be accessible for 5 days after the posting date of the notice, and it included instructions on how to connect to the account containing the survey. Given the short, 5-day window of opportunity to participate, and that not every potential respondent willing to participate necessarily read the newsgroup regularly, we consider 72 respondents to be a quite respectable rate of return. Also, the number of daily contributions may be a better indicator of the number of active members, and hence of the number of likely respondents, than would the total number of subscribers (this issue is something that must be examined further). Using this number ( 12 contributions per day for 5 days) as the basis upon which to estimate return rate suggests that we might expect 60 responses. Receiving 72 respondents over 5 days would therefore be a very good rate of return for the volunteer notice. It must, however, be kept in mind that, due to the technique we used to collect data, there was no way to determine if the subjects were active contributors.

\section{Procedure and Materials}

The introductory psychology students anonymously completed a pencil-and-paper version of a questionnaire on multiple-choice scantron sheets in groups of 1 to 8 . The researcher produced a pile of surveys in unmarked envelopes and shuffled them in the presence of the subjects, who then selected their envelopes from anywhere within the pile. After completing the survey, the subjects placed their surveys back into the unmarked envelopes and returned them to any position within a pile of already completed surveys.

The survey began with the following:

We are doing an independent survey in the field of human sexuality All the questions refer to you and your sexual thoughts and fantasies. This survey should take approximately 30 minutes to complete. After completing the survey please return it to the investigator. Please be as honest as possible. Remember, this is not a test and there are no trick questions.

Description: Sexual fantasies may be externally provoked or internally generated. That is sexual fantasies may be stimulated by something you see, read or hear in the environment, or they may occur spontaneously.

The survey contained 7 demographic items and 28 items asking for fantasy-related information. Two demographic items permitted subject-generated responses (i.e., "other - please describe"). The responses were made on scantron sheets to minimize the visibility of answers and to ensure confidentiality. The responses were later read into a computer file for further analysis. 
Internet subjects used the Telnet protocol to connect to a 3100 VAX Model 80 mainframe computer running the VMS (version 5.5) operating system. Third-party software (TGV Multinet software, version 3.3) containing the Telnet protocol was used because the VMS operating system did not support direct Internet access. The VAX supported VT100, VT220, VT320, and VT420 terminal emulators.

The Internet version of the survey was run within a captive account. We disabled normal break keys ( $\mathrm{Ctrl}-\mathrm{y}$ and $\mathrm{Ctrl}-\mathrm{z}$ ) in the account's user profile. Another standard break key $(\mathrm{Ctrl}-\mathrm{c})$ could not be disabled in this profile but was disabled within the survey program. The host computer ignored responses other than those enumerated in the survey or automatically disconnected the subject. After subjects had read the consent form and typed the password to indicate their consent, a standard login.com file (written in DCL) automatically started the survey (written in VAX basic, version 3.3).

The survey consisted of the same series of multiple-choice items as the pencil-and-paper questionnaire. Each item was individually presented on the screen, and subjects were prompted for their responses. Every item included an option to withdraw from the research.

The time lag between the host computer and subjects was a problem. Preliminary work had indicated that subjects often pressed the Enter/Return keys several times in rapid succession if the host computer's response was slow or it did not appear to be responding. As a result, they often failed to respond to several items or the responses did not accurately reflect the answer to that particular item. To minimize this problem, before presenting the next item the software prompted subjects to confirm their responses.

After completing the survey, subjects read a debriefing notice containing the rationale of the research and the e-mail address of the experimenter. The host computer automatically terminated the connection and appended the data to a master data file maintained in another account. Each data file contained the data from the survey, the time subjects logged out, the time it took to complete the survey, the unique file name, and the domain address of the subject.

\section{RESULTS}

The survey contained seven demographic variables: age, gender, sexual orientation, marital status, ethnicity, education, and religiosity. Not every subject completed every item. However, the number of nonresponses on any particular item was low and appeared to be equally distributed between the two populations. A chi-square analysis was performed on each demographic variable to determine if the two samples differed. The results are summarized in Table 1.

The two samples did not differ significantly on five of the seven variables: sexual orientation, marital status, eth- nicity, education, and religiosity. However, the analyses revealed highly significant differences on the gender and age variables. A larger percentage of the males in the survey came from the Internet sample $(83 \%)$ and a greater percentage of the female subjects who participated in the survey came from the campus sample $(70 \%)$. Both populations had gender disparities. The majority of subjects from the Internet sample were male $(74 \%)$, and the campus sample was primarily female $(80 \%)$. There are several possibilities for why Internet respondents were primarily male. Males may be more heavily represented in the Internet population than in the general population; females using the Internet may be less likely to agree to participate in this particular research project, or perhaps few females read that particular Usenet group. More research to distinguish among these possibilities and others is needed.

On the age variable, $47 \%$ of the subjects below the age of 30 were from the Internet sample; $89 \%$ of the subjects over the age of 30 were from the Internet sample. The majority of subjects in both samples were below the age of 30 . The campus sample had a larger percentage of subjects below the age of 30 than did the Internet sample $(95 \%$ and $65 \%$, respectively). This difference in age distribution suggests that there may be a greater range of ages available in Internet samples than in student samples.

These results provide some insight into the generalizability of data collected from Internet subjects. The Internet sample was similar to a traditional sample in ethnicity, sexual orientation, marital status, and religiosity, suggesting that Internet samples are as representative as traditional student samples. Differences on the age variable suggest that Internet samples are better than traditional samples because they contain a broader range of ages upon which to draw. However, more research, using a broader range of demographic variables and newsgroups, needs to be done to replicate these findings before it will be possible to establish firmly that any data collected from Internet subjects are generalizable.

The second point that supports the argument that Internet data are generalizable is our replication of the research conducted by Ellis and Symons (1990), using different sample populations. Our samples differed from Ellis and Symons's on several dimensions. First, we sampled Internet subjects and introductory psychology students,

Table 1

Results of Chi-Square Analyses on the Percentage of Subjects in the Seven Demographic Variables as a Function of Subject Population

\begin{tabular}{|c|c|c|c|c|c|c|c|c|}
\hline \multirow[b]{2}{*}{ Variable } & \multicolumn{4}{|c|}{ Sample Population } & \multirow[b]{2}{*}{$d f$} & \multirow[b]{2}{*}{$N$} & \multirow[b]{2}{*}{$\chi^{2}$} & \multirow[b]{2}{*}{$p$} \\
\hline & Internet & $n$ & Campus & $n$ & & & & \\
\hline Age $(\leq 30 />30)$ & $65 / 35$ & 72 & $95 / 5$ & 56 & 1 & 128 & 15.89 & $<.001$ \\
\hline Gender (male/female) & $74 / 26$ & 72 & $20 / 80$ & 56 & 1 & 128 & 36.70 & $<.001$ \\
\hline Sexual orientation (hetero/homosexual) & $94 / 06$ & 70 & $98 / 2$ & 55 & 1 & 125 & 1.22 & .270 \\
\hline Marital status (single/not single*) & $42 / 58$ & 72 & $55 / 45$ & 56 & 1 & 128 & 2.37 & .120 \\
\hline Ethnicity (minority/nonminority: white) & $14 / 86$ & 72 & $15 / 85$ & 53 & 1 & 125 & 0.04 & .850 \\
\hline Education ( $\leq$ associate bachelors $\geq \geq$ bachelors $\dagger$ ) & $17 / 83$ & 72 & $15 / 85$ & 55 & 1 & 127 & 0.11 & .750 \\
\hline Religiosity (religious/nonreligious) & $58 / 42$ & 69 & $56 / 44$ & 52 & 1 & 121 & 0.06 & .810 \\
\hline
\end{tabular}


whereas Ellis and Symons sampled upper level nonpsychology students. Second, both of our samples were selfselected, whereas Ellis and Symons's sample was not. They distributed their survey to entire classes selected in advance. Our method of administering the survey also differed in that Internet subjects individually completed our survey in a networked environment and not in a room with many other subjects present. Obtaining similar patterns of responses, despite these differences in sample population, subject selection, survey administration, and testing environments, strongly argues in favor of the generalizability and validity of data collected from Internet subjects and hence the utility of using the Internet as an alternative or supplemental source of subjects.

This discussion of data validity does not warrant a full discussion of the results of this survey. However, we will discuss six questionnaire items to illustrate that the patterns of responding were comparable.

We analyzed our data in two stages. In the first, we made within-sex comparisons on six items, comparing the responses of Internet-sample males with those of campussample males and the responses of Internet-sample females with those of campus-sample females.

Item 1 used a rating scale of 0 to 7 with the alternatives of Never, Once a week, Once a day or less, About once a day, 2 or 3 times a day, 4 to 6 times a day, 7 to 10 times a day, More than 10 times a day. It asked, "Approximately how often do you have sexual fantasies?"

Items 2 and 3 used a rating scale of 0 to 3 with the alternative answers of Very important, Somewhat important, Not very important, or Not at all important. Item 2 asked, "How important is caressing and non-genital touching in your sexual fantasies?" Item 3 asked, "How important is the emotional setting (such as mood and ambiance) in your sexual fantasies?"

The final three items were categorical. Item 4 asked, "During sexual fantasy, do you focus more on (1) the sexual act itself, (2) the physical characteristics of your fantasized partner, (3) the personal or emotional characteristics of your fantasized partner, (4) your own physical or emotional response." Item 5 asked, "Which is a more important part of your sexual fantasies?" and had the options of "(1) visual images, (2) touching, 3) neither." Item 6 asked, "Are your fantasies typically about: (1) someone who you are, or have been, romantically/sexually involved with, (2) someone (even if they are made up) who you would like to become romantically involved with, (3) someone (even if they are made up) who you would like to become sexually involved with, (4) none of the above."

A total of 64 males responded to these items, 53 from the Internet sample and 11 from the campus sample (with the exception of Item 4, to which 10 campus males responded). The mean responses for Items $1-3$ are shown in Table 2. Two-tailed $t$ tests did not reveal any significant differences between the two samples. A chi-square analysis on Items 4-6 also did not reveal significant differences between Internet and campus responses [Item 4 , $\chi^{2}(3)=1.85 ;$ Item $5, \chi^{2}(2)=1.00 ;$ Item $6, \chi^{2}(3)=$ 3.76 ; all $p \mathrm{~s}>.28]$.
A total of 63 females responded to these items, 19 from the Internet sample and 44 from the campus sample (with the exception of Item 4 , to which 43 campus females responded, and Item 5, to which 18 Internet females responded). The mean responses to Items 1-3 are shown in Table 2. Two-tailed $t$ tests did not reveal any significant differences between the responses of each sample. Chisquare analyses on Items 4-6 also did not reveal significant differences between Internet and campus responses [Item $4, \chi^{2}(3)=6.50 ;$ Item $5, \chi^{2}(2)=2.37$; Item 6, $\chi^{2}(3)=2.83 ;$ all $\left.p s>.08\right]$.

The second stage of analysis involved comparing male and female responses to see if our results were comparable to Ellis and Symons's. Since the analyses above showed that patterns of responding within each sex were similar between the two samples and that all items in both samples exhibited patterns of responding in the predicted direction, we collapsed across samples for each sex and analyzed the data for comparison with Ellis and Symons's results.

For Item 1, Ellis and Symons obtained means of 1.97 $(S D=1.16)$ for females and $3.22(S D=1.46)$ for males $[t(304)=8.33, p<.001]$. We obtained means of 2.58 $(S D=1.44)$ for females and $3.52(S D=1.31)$ for males $[t(204)=4.87, p<.001]$.

For Item 2, Ellis and Symons obtained means of 0.49 $(S D=.81)$ for females and $1.04(S D=.83)$ for males $[t(305)=5.29, p<.001]$. We obtained means of 0.59 $(S D=.88)$ for females and $1.00(S D=.81)$ for males $[t(205)=3.44, p<.001]$. For Item 3, Ellis and Symons obtained means of $0.61(S D=.68)$ for females and 0.96 $(S D=.81)$ for males $[t(302)=4.09, p<.001]$. We obtained means of $0.66(S D=.85)$ for females and 1.22 $(S D=.94)$ for males $[t(205)=4.41, p<.001]$.

A chi-square analysis was performed on Items 4-6. As shown in Table 3, the percentages and significance values obtained by Ellis and Symons and us are comparable for these three items.

\section{DISCUSSION}

Overall, although our samples differed from Ellis and Symons's (1990) sample on several dimensions, these differences had little, if any, effect on the results. The analy-

Table 2

Mean ( $\pm 1 S D)$ Male and Female Responses

From Internet and Campus Samples to Three Items

\begin{tabular}{|c|c|c|c|c|c|c|c|}
\hline \multirow[b]{3}{*}{ Item } & \multicolumn{4}{|c|}{ Response } & \multirow[b]{3}{*}{$t$} & \multirow[b]{3}{*}{$d f$} & \multirow[b]{3}{*}{$p$ (2-tailed) } \\
\hline & \multicolumn{2}{|c|}{ Internet } & \multicolumn{2}{|c|}{ Campus } & & & \\
\hline & $M$ & $\overrightarrow{S D}$ & $M$ & $S D$ & & & \\
\hline \multicolumn{8}{|c|}{ Male } \\
\hline 1 & 0.89 & 0.82 & 1.33 & 0.51 & 1.84 & 62 & .07 \\
\hline 2 & 0.88 & 0.80 & 1.18 & 0.87 & 1.10 & 62 & .27 \\
\hline 3 & 3.85 & 1.32 & 3.82 & 1.17 & 0.07 & 62 & .94 \\
\hline \multicolumn{8}{|c|}{ Female } \\
\hline 1 & 0.74 & 1.04 & 0.59 & 0.84 & 0.59 & 61 & .56 \\
\hline 2 & 0.58 & 0.84 & 0.55 & 0.66 & 0.17 & 61 & .87 \\
\hline 3 & 2.89 & 1.79 & 2.30 & 1.44 & 1.41 & 61 & .17 \\
\hline
\end{tabular}


ses above support two other conclusions. First, Internet subjects can be used as a primary subject population, from which all the subjects are drawn. Second, the Internet can also serve as a supplemental subject pool. When researchers have difficulty in locating sufficient numbers of subjects in their local areas, an Internet sample can reasonably be combined with the local sample for a single analysis.

There is also the issue of uncontrolled settings. For obvious reasons, there will be little consistency of environmental settings among Internet subjects. Such subjects might be at home in a darkened room or in a large and noisy lab when they participate. Although these variations did not affect the results of our survey, it is conceivable that they could affect other types of research. Information from subjects on their environments and their interface software and configurations would be useful in determining the sensitivity of experimental procedures to these types of variations. If experiments are sensitive to particular kinds of environmental variations, researchers can specify the conditions under which the experiment should be run.

\section{Self-Selection Bias}

Perhaps the most important difference between Ellis and Symons's sample and ours is that our sample was selfselected. This issue warrants further discussion. Subjects are active decision makers, and this active choice is a potential source of unmeasurable systematic error. The decision of subjects to not participate might be a conscious

Table 3

Comparison of Responses Obtained by Ellis and Symons With Responses Obtained by Smith and Leigh to Items 4-6

\begin{tabular}{|c|c|c|c|c|}
\hline \multirow[b]{2}{*}{ Study } & \multicolumn{4}{|c|}{ Option } \\
\hline & 1 & 2 & 3 & 4 \\
\hline \multicolumn{5}{|c|}{ Item 4} \\
\hline \multicolumn{5}{|c|}{ Ellis and Symons $\dagger\left(\chi^{2}=34.33, d f=3\right)$} \\
\hline Females & $59 \%$ & $25 \%$ & $9 \%$ & $7 \%$ \\
\hline Males & $28 \%$ & $38 \%$ & $29 \%$ & $5 \%$ \\
\hline \multicolumn{5}{|c|}{ Smith and Leigh* $\left(\chi^{2}=13.76, d f=3\right)$} \\
\hline Females & $51 \%$ & $21 \%$ & $17 \%$ & $11 \%$ \\
\hline Males & $26 \%$ & $19 \%$ & $47 \%$ & $8 \%$ \\
\hline \multicolumn{5}{|c|}{ Item 5} \\
\hline \multicolumn{5}{|c|}{ Ellis and Symons $\dagger\left(\chi^{2}=43,47, d f=1\right)$} \\
\hline Females & $43 \%$ & $57 \%$ & & \\
\hline Males & $81 \%$ & $19 \%$ & & \\
\hline \multicolumn{5}{|c|}{ Smith and Leight $\left(\chi^{2}=11.07, d f=2\right)$} \\
\hline Females & $36 \%$ & $57 \%$ & $7 \%$ & \\
\hline Males & $66 \%$ & $30 \%$ & $4 \%$ & \\
\hline \multicolumn{5}{|c|}{ Item 6} \\
\hline \multicolumn{5}{|c|}{ Ellis and Symons $\dagger\left(\chi^{2}=66.64, d f=3\right)$} \\
\hline Females & $12 \%$ & $13 \%$ & $41 \%$ & $34 \%$ \\
\hline Males & $20 \%$ & $50 \%$ & $16 \%$ & $13^{\circ}$ \\
\hline \multicolumn{5}{|c|}{ Smith and Leight $\left(\chi^{2}=16.56, d f=3\right)$} \\
\hline Females & $23 \%$ & $8 \%$ & $27 \%$ & $42 \%$ \\
\hline Males & $41 \%$ & $24 \%$ & $19 \%$ & $16 \%$ \\
\hline
\end{tabular}

Note-Ellis and Symons's data are from "Sexual Differences in Sexual Fantasy: An Evolutionary Approach," by B. Ellis \& D. Symons, 1990, Journal of Sex Research, 27, p. 539. Copyright 1990 by the Society for the Scientific Study of Sex. Adapted with permission. ${ }^{*} p<.005 . \quad{ }^{\dagger} p<.001$. decision to preserve their self-esteem or to protect themselves against the perceived purposes of the experimenter; or potential subjects might not consider such participation as making efficient use of their own time (Bowden, 1986). This potential exists for subjects solicited from both the Internet and traditional sources.

There are two points to make on this issue. First, it is difficult to ensure that the sample is unbiased. Introductory psychology students do not represent an unbiased sample of the overall population. They are a very narrow sample of the overall population in terms of age and profession. They tend to be better educated, to come from higher socioeconomic-status families, and to be between the ages of 18 and 27. Most researchers consider this level of bias acceptable, or they use established techniques to screen for potential bias (see Biggar \& Melbye, 1992; Bowden, 1986). Second, our Internet sample had a broader age distribution than does a typical student sample. Therefore, at least on this dimension, a case can be made that an Internet sample would be less biased than are traditional samples. However, because using the Internet as a source of subjects is only in the early stages of development, the prudent course would be to compare traditional samples with Internet samples to establish data reliability and replicability estimates.

In this section, we have discussed the important issue of data validity. The ability to recruit subjects from the Internet is of little use if the data collected are only marginally useful. We have discussed several procedures researchers could use to minimize the risk of subject fraud, whether data collected from Internet subjects would be generalizable, and the potential problem of a self-selection bias. The data and discussion presented in this section support the argument that data are generalizable. However, we also have emphasized the importance of establishing comparability between traditional subject samples and Internet samples.

\section{Current Limitations and Future Potential}

Just as every type of research cannot be performed on desktop computers or with pencil and paper, there are limitations to conducting research on the Internet. The current primary limitation to conducting research with subjects connected to a remote host is timing (i.e., reaction times and presentation rates). The response time between a remote host and a subject depends on many factors that are experimentally uncontrollable. These include the amount of traffic on the Internet, the number of intermediary computers between the subject and the host, and the subject's distance from the host. Response times between subjects and the host computer continually change as various users connect to and disconnect from different hosts on the network, even during an interactive session with a remote host. There are several potential solutions to this problem. Hewson et al. (1996) suggest placing experimental programs on an FTP (file transfer protocol) site and permitting volunteers to download the software to run on their individual machines. Including protocols in the software to forward data files automatically to the 
researcher can also decrease demands on the volunteer, making this strategy less daunting to less proficient users of the Internet. This technique, however, is subject to the same limitations as broadly distributing a survey to newsgroups (discussed earlier). Another potential solution is a new protocol called Java. Java is architecture neutral, meaning that software developed using this protocol will run on any computer. This protocol permits real-time running of active, dynamic simulations on the Internet (see Gosling \& McGilton, 1995). A protocol called virtual reality markup language (VRML) holds similar possibilities (Kieley, 1996).

Other examples of experiments not currently easily conducted on the Internet include experiments requiring face-to-face interactions between the experimenter and the subject, longitudinal studies, or research that requires the exchange of physical items. However, as the Internet's capabilities continue to expand, some of these limitations also may be overcome. For example, there is software and relatively inexpensive hardware currently available that permit individuals to view one another using the Internet. Although this technology is not yet widespread and is still unrefined (motion is presented as a series of still images rather than true full motion), it does hold the promise of overcoming some of the limitations relating to face-toface interactions. So, these current limitations should not deter researchers from using the Internet as a research environment. As mentioned above, there remain many types of experiments that are currently possible and many more possibilities are likely in the future.

There are three other areas where the Internet could be developed. The first is in the use of the Internet for traditional research. Almost any experiment capable of running on a desktop computer can be run from a remote site. Subjects can log-on to a remote host computer and complete an experiment as if they were completing it on a local, desktop PC. Just like designing a between, within, or mixed experimental design to run on a PC, running an experiment on the Internet merely requires writing programming code to assign subjects to the appropriate conditions. This is easily accomplished on the Internet. For example, if random assignment of subjects is necessary, assign the first subject to Group A, the second to Group B, the third to Group C, the fourth to Group A, and so on. This simple method of assigning subjects to conditions will be essentially random. Alternatively, if subjects need to be assigned to different conditions on the basis of a particular characteristic (e.g., age, sex, math skills), subjects can provide that information before presentation of the experimental material. Considering this information, software can assign subjects to appropriate conditions. For example, if subjects are to be assigned to an experimental group on the basis of mathematical ability, subjects could complete a math pretest. Subjects with a poor score could be assigned to the poor-math-skills group. Subjects with a good score could be assigned to a good-math-skills group.

With subjects assigned to appropriate groups, there still remains the task of data collection. As mentioned ear- lier, experiments dependent on time are not very viable when a remote host is used as the site of the experiment. However, experiments that require immediate feedback or that require subjects to interact actively with experimental protocols are easily accommodated using a remote host connection as the site of the experiment. To illustrate this capability, we will use a categorization task as an example. Vokey and Brooks (1992) examined how the salience of item knowledge influenced the learning of artificial grammars. Subjects were asked to memorize several lists of letter strings at their own pace. The letter strings were consistent with an arbitrary set of grammatical rules. During the test phase, the subjects were informed that the letter strings they had just studied were consistent with a complex series of rules. They were then asked to judge whether new letter strings were grammatically consistent with items from the study list. This type of study could easily be conducted using the methods outlined here. During the study phase, subjects could study the items and then send a response to the host computer to indicate their readiness to receive the next list of letter strings. During the test phase, items could be presented serially or in a list, with subjects indicating whether or not the items were consistent and then indicating when the host computer needed to present the next series of items. The subject's response, which controls the responses of the host, can be stored in a data file on the remote host. Another procedure that may require the host computer to interact with a subject might involve conditional stimulus sequences where a subject's response determines the sequence of subsequent stimuli (e.g., keep presenting one type of stimulus until correctly identified and count the number of errors). In these kinds of procedures, the remote host computer can be used to collect real-time data and respond interactively with subjects.

Designing and running different experimental designs on the Internet with subjects from remote locations does not fully address the Internet's potential as a research environment. There still remains the question of the specific types of experiments that can be run. Perhaps the best way to address this issue is to mention possible types of experimental materials and procedures. This provides an idea of the flexibility and range of experiments without limiting the scope to specific research domains.

A large portion of the Internet is text based. Experiments that use text as experimental material are easily adapted to be run using Internet subjects. These include, but are not limited to, the presentation of words, letters, phrases, stories, or numbers. However, not every researcher uses text as experimental material. Images, color, sound, and motion are also common. Obviously, experiments using these types of materials cannot be performed on text-based systems. However, the Internet does support these types of materials. The World-Wide Web (WWW), a rapidly expanding portion of the Internet, supports all these aspects with web-browsing software (e.g., Netscape and Mosaic). The research possibilities of the WWW can be further enhanced by using the common gateway interface (CGI) 
protocol. CGI is a scripting language that allows the execution of programs residing on the host computer, permitting stimuli and data to travel between users and the host on the WWW. Its numerous capabilities include data parsing, scoring tests, and electronic conferencing (Kieley, 1996). These capabilities are clearly useful for research on the Internet.

Finally, the Internet can be used to improve educationdelivery techniques. Education delivery is changing (Markwood, 1994). Universities and colleges across British Columbia are establishing interactive television (ITV) networks, enabling instructors to deliver educational services from a distant single site (Harby, Quinlan, \& Roy, 1994). Other provinces and American states and European countries are establishing similar networks (Kershaw, 1993, 1995; Mason, 1993). These networks allow students to receive two-way interactive instruction from instructors at a different geographical location. This capability is increasing the numbers of students opting to receive portions of their postsecondary education closer to home. These changes have several implications. Students physically present on campus have the opportunity to participate in experiments (generally considered a beneficial educational experience). Students taking courses via ITV do not have this opportunity and hence are at a disadvantage. Instructors have a responsibility to ensure that these students receive educational opportunities that are similar to those of students physically attending a campus. Using the Internet is one way to achieve this goal by permitting physically distant students to participate in some forms of experimentation and, therefore, to enhance their educational experience.

\section{Conclusions}

This paper has proposed that the Internet is a useful research environment and a potential source of research subjects. It has presented a rationale for why some researchers might consider using the Internet for this purpose and has outlined some of the Internet's characteristics that permit researchers to accomplish this goal. It has also addressed some ethical issues. We have discussed some guidelines for conducting research on the Internet and the operationalization of these guidelines. To support the argument that data collected from Internet subjects can be useful and valid, we compared an Internet sample and an introductory psychology sample on several demographic variables and replicated previously published research. Finally, we discussed some limitations of using the Internet for research and some potentials. By addressing these issues, we have taken an important step in developing the Internet as a useful research tool.

\section{REFERENCES}

BigGaR, R. J., \& MELbye, M. (1992). Responses to anonymous questionnaires concerning sexual behavior: A method to examine potential biases. American Journal of Public Health, 82, 1506-1512.
BORDIA, P. (1996). Studying verbal interaction on the Internet: The case of rumor transmission research. Behavior Research Methods, Instruments, \& Computers, 28, 149-151.

BOWDEN, R. J. (1986). Self-selection biases in correlational studies based on questionnaires. Psychometrika, 51, 313-325.

Castellan, N. J., JR. (1991). Computers and computing in psychology: Twenty years of progress and still a bright future. Behavior Research Methods, Instruments, \& Computers, 23, 106-108.

Ellis, B. J., \& Symons, D. (1990). Sex differences in sexual fantasies: An evolutionary psychological approach. Journal of Sex Research, 27, 527-555.

Esposito, J., AGaRd, E., \& Rosnow, R. (1984). Can confidentiality of data pay off? Personality \& Individual Differences, 5, 744-745.

GoslinG, J., \& MCGILTON, H. (1995). The Java language environment: A white paper. Sun Microsystems, Inc. Available WWW: http://java. sun.com/whitePaper/java-whitepaper-1.html

HaRby, A., Quinlan, M., \& RoY, R. (1994). Interactive video conferencing in British Columbia. (Available from the Standing Committee on Educational Technology, c/o Centre for Curriculum \& Professional Development, 5th Floor, 1483 Douglas St., Victoria, BC, Canada V8W 3K4)

Hewson, C. M., Laurent, D., \& Vogel, C. M. (1996). Proper methodologies for psychological and sociological studies conducted via the Internet. Behavior Research Methods, Instruments, \& Computers, 28, 186-191.

Hoffman, R., \& MacDonald, J. (1993). Using HyperCard and Apple events in a network environment: Collecting data from simultaneous experimental sessions. Behavior Research Methods, Instruments, \& Computers, 25, 114-126.

Huff, C. W., \& Rosenberg, J. (1989). The on-line voyeur: Promises and pitfalls of observing electronic interaction. Behavior Research Methods, Instruments, \& Computers, 21, 166-172.

KERSHAw, A. (1993). The challenge of the place bound student: A potential model for the delivery of post-secondary education in the $U C C$ region and beyond. Unpublished manuscript, University College of the Cariboo, Kamloops, British Columbia, Canada.

KERSHAW, A. (1995, January). Distance delivery technologies: Learning models and optimum applications. Paper presented to the British Columbia Interactive Video User's group, Vancouver.

KIELEY, J. M. (1993). Integrating remotely accessible information services into psychology instruction and research. Behavior Research Methods, Instruments, \& Computers, 25, 287-294.

KIELEY, J. M. (1996). CGI scripts: Gateways to World-Wide Web power. Behavior Research Methods, Instruments, \& Computers, 28, 165-169.

KROL, E. (1992). The whole Internet. Sebastapol, CA: O'Reilly \& Associates.

LESGOLD, A. (1991). Research methodology in the postinformatic age. Behavior Research Methods, Instruments, \& Computers, 23, 109-111.

MARKWOOD, R. A. (1994). Computer tools for distance education. In B. Willis (Ed.), Distance education: Strategies and tools (pp. 199-211). Englewood Cliffs, NJ: Educational Publications.

Mason, R. (1993). Computer conferencing and the new Europe. In L. M. Harasim (Ed.), Global networks: Computers and international communication (pp. 199-220). Cambridge, MA: MIT Press.

Polson, P. G., Milier, J. R., \& Karat, J. (1981). Psychological experimentation through direct connection of the subject interface to a VAX. Behavior Research Methods, Instruments, \& Computers, 13, 189-191.

REID, B. (1994, July). Usenet readership summary report. Network Measurement Project at the DEC Network Systems Laboratory in Palo Alto. Available Usenet: news.lists

VOKEY, J. R., \& BRoOKs, L. R. (1992). Salience of knowledge in learning artificial grammars. Journal of Experimental Psychology, 18, 328-344.

YOST, M., \& BREMNER, F. (1985). The use of computer networks in data gathering and data analysis. Behavior Research Methods, Instruments, \& Computers, 17, 157-159.

(Manuscript received August 10, 1995; revision accepted for publication August 22, 1996.) 